\title{
DATA NETWORK MODELS OF BURSTINESS
}

\author{
BERNARDO D'AURIA AND SIDNEY I. RESNICK
}

\begin{abstract}
We review characteristics of data traffic which we term stylized facts: burstiness, long range dependence, heavy tails, bursty behavior determined by high bandwidth users, dependence determined by users without high transmission rates. We propose an infinite source Poisson input model which accounts for traffic in adjacent time slots. This model has the ability to account for many of the stylized facts.
\end{abstract}

\section{INTRODUCTION}

Measurements on data networks often show empirical features that are surprising by the standards of classical queueing and telephone network models. Measurements often consist of data giving bit-rate or packet rates. This means that a window resolution is selected (for example, 10 seconds, 1 second, 10 milliseconds, 1 millisecond, ... ) and the number of bits or packets in adjacent windows or slots is recorded. Significant examples include Duffy et al. (1993), Leland et al. (1993), Willinger et al. (1995, 1997).

Despite the fact that collected data is for time slots of modest size, many of the theoretical attempts to create models to explain the empirical observations concentrate on large time scales and cumulative traffic over large time intervals. See, for example, Heath et al. (1998), Kaj and Taqqu (2004), Konstantopoulos and Lin (1998), Levy and Taqqu (2000), Maulik and Resnick (2003), Mikosch et al. (2002), Taqqu et al. (1997). For such models, it is difficult to find agreement with many existing data sets (Guerin et al. (2003)).

Many network data sets exhibit distinctive properties, which in analogy with empirical finance, we will term stylized facts:

- Heavy tails abound (Leland et al. (1994), Willinger et al. (1998), Willinger and Paxson (1998), Willinger (1998) ) for such things as file sizes (Arlitt and Williamson (1996), Resnick and Rootzén (2000)), transmission rates, transmission durations (Maulik et al. (2002), Resnick (2003)).

- The number of bits or packets per slot exhibits long range dependence across time slots (eg, Leland et al. (1993), Willinger et al. (1995). There is also a perception of self-similarity as the width of the time slot varies across a range of time scales exceeding a typical round trip time.

- Network traffic is bursty with rare but influential periods of very high transmission rates punctuating typical periods of modest activity.

Burstiness, a somewhat vague concept, is an important feature of traffic because of the sudden peak loads it introduces to the network. Attempts to understand this phenomenon empirically (Sarvotham et al. (2005)) use the $\alpha / \beta$ decomposition of users where the $\alpha$-users transmit large files at very high rate and $\beta$-users transmit the rest. Alternative language creates a dichotomy between mice and elephants (Azzouna et al. (2004)) depending on whether a file is typical or very large. Some stylized facts suggested by the stimulating empirical study (Sarvotham et al. (2005)) include:

- Large files over fast links contribute to $\alpha$-traffic. The $\alpha$-component consitutes a small fraction of total workload but is entirely responsible for burstiness. Often a single dominent high-rate connection causes a burst.

Key words and phrases. Bursty traffic, M/G/ $\infty$ input model, infinite source Poisson model, network modelling, limit distributions, Lévy processes, Gaussian limits.

Sidney Resnick's research was partially supported by NSF grant DMS-0303493. Much of this research took place during Sid Resnick's visit to Eurandom during May-June 2005 and grateful acknowledgement for support and hospitality is made. The final formulation of the results and the majority of the writing took place during Sid Resnick's July 2005 visit to the Mathematical Sciences Institute, The Australian National University, Canberra ACT, Australia. Support from the Centre of Excellence for Mathematics and Statistics of Complex Systems is gratefully acknowledged. 
- Most of the dependence structure across time slots is carried by the $\beta$-traffic. The long range dependence structure of the $\beta$-traffic approximates that of the complete traffic.

- The quantity of traffic in a time window is distributionally approximated by the normal distribution when there is high levels of aggregation across users and heavy loading. $\beta$-traffic is much more likely to appear Gaussian than $\alpha$-traffic.

Owing to measurements being taken for fixed time slots, we begin our attempt to provide models explaining the empirically observed stylized facts by modeling the quantity of data in adjacent time slots of length $\delta$. Then to get approximations and to provide a clarified asymptotic picture of behavior, we let $\delta \rightarrow 0$ and see what limits exits. In particular we seek a model that explains the origins of burstiness.

\section{Model Description}

The model for data traffic generation is a slight modification of the $M / G / \infty$ input or infinite source Poisson model as we assume that also the transmission rates are random. (See also, Maulik et al. (2002).) We assume that a homogeneous Poisson process on $\mathbb{R}$ with points $\left\{\Gamma_{k}\right\}$ activates data transmission sessions. The parameter or rate of the Poisson process is $\lambda=\lambda(\delta)$, and each transmission activation time $\Gamma_{k}$, has associated three additional quantities $\left(R_{k}, L_{k}, F_{k}\right)$. These three quantities have the following physical interpretations:

- $\mathrm{R}$ - Rate of the transmission,

- L - Duration of the transmission,

- F - Size of the transmitted file.

Obviously these three quantities are related by the following relation

$$
F=R \cdot L \text {. }
$$

We assume the marks $\left\{\left(R_{k}, L_{k}, F_{k}\right),-\infty<k<\infty\right\}$ are iid and independent of $\left\{\Gamma_{k}\right\}$. The univariate marginal distributions of the triple are

$$
G(x)=P\left[F_{1} \leq x\right], \quad F_{R}(x)=P\left[R_{1} \leq x\right], \quad F_{L}(x)=P\left[L_{1} \leq x\right] .
$$

We suppose that all three distributions are heavy tailed

$$
\bar{G}(x)=x^{-\alpha_{F}} L_{F}(x), \quad \bar{F}_{R}(x)=x^{-\alpha_{R}} L_{R}(x), \quad \bar{F}_{L}(x)=x^{-\alpha_{L}} L(x),
$$

where $L_{F}, L_{R}, L$ are all slowly varying and we assume all three tail parameters satisfy

$$
1<\alpha_{F}, \alpha_{R}, \alpha_{L}<2
$$

There is empirical evidence justifying these assumptions. See Azzouna et al. (2004), Campos et al. (2005), Guerin et al. (2003), Heffernan and Resnick (2005), Leland et al. (1994), Maulik et al. (2002), Park and Willinger (2000), Resnick (2003, 2004), Riedi and Willinger (2000), Sarvotham et al. (2005), Willinger et al. (1995).

With these assumptions, the counting function of the points $\left\{\left(\Gamma_{k}, R_{k}, L_{k}, F_{k}\right)\right\}$

$$
N=\sum_{k} \epsilon_{\left(\Gamma_{k}, R_{k}, L_{k}, F_{k}\right)}
$$

on $\mathbb{R} \times[0, \infty)^{3}$ is a Poisson random measure with mean measure

$$
\lambda d s P\left[\left(R_{1}, L_{1}, F_{1}\right) \in(d r, d l, d u)\right]=: \mu^{\#}(d s, d r, d l, d u) .
$$

See, for example, Kallenberg (1983), Neveu (1977), Resnick (1987, 1992).

For a time window of length $\delta$, we will consider weak limits of the process

$$
\mathbf{A}(\delta):=\{A(k \delta,(k+1) \delta],-\infty<k<\infty\}
$$

as $\delta \downarrow 0$. Here $A(k \delta,(k+1) \delta]$ represents the total mount of work inputted to the system in the $k$-th time slot $(k \delta,(k+1) \delta]$. We will define this precisely for $k=0$ and the definitions for the other values of $k$ will be obvious by analogy.

Distinguish 4 disjoint regions in $\mathbb{R} \times[0, \infty)^{3}$ :

$$
\mathcal{R}^{>0,1}=\{(s, r, l, u): 0<s \leq \delta, 0<s+l \leq \delta\},
$$




$$
\begin{aligned}
& \mathcal{R}^{>0,2}=\{(s, r, l, u): 0<s \leq \delta, s+l>\delta\}, \\
& \mathcal{R}^{<0,1}=\{(s, r, l, u): s<0,0<s+l \leq \delta\}, \\
& \mathcal{R}^{<0,2}=\{(s, r, l, u): s<0, s+l>\delta\} .
\end{aligned}
$$

Region $\mathcal{R}^{>0,1}$ corresponds to sessions which start and end in $(0, \delta]$ while the region $\mathcal{R}^{>0,2}$ describes sessions starting in $(0, \delta]$ but ending subsequent to $\delta$. Region $\mathcal{R}^{<0,1}$ has sessions starting prior to time 0 and ending in $(0, \delta]$ while $\mathcal{R}^{<0,2}$ has sessions initiated prior to 0 and ending subsequent to $\delta$. See Figure 1.

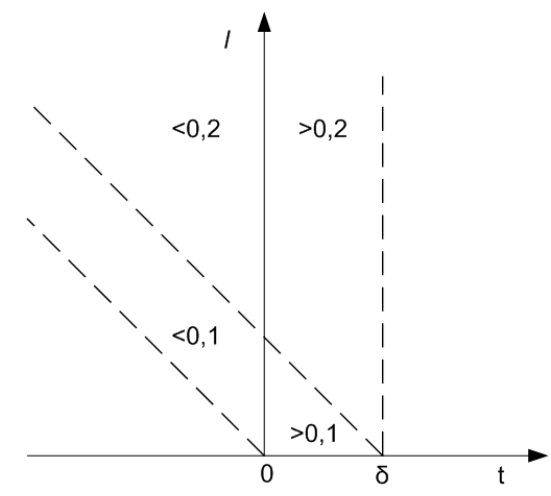

FiguRE 1. Four regions.

Corresponding to this decomposition of regions, if we restrict the Poisson random measure to the 4 regions, we get 4 independent Poisson processes:

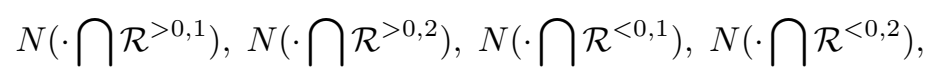

and we use these to express $A(0, \delta)=: A(\delta)$ as the sum of 4 independent contributions:

$$
A(\delta)=A^{>0,1}(\delta)+A^{>0,2}(\delta)+A^{<0,1}(\delta)+A^{<0,2}(\delta),
$$

where

$$
\begin{aligned}
& A^{>0,1}(\delta)=\sum_{k} R_{k} L_{k} 1_{\left.\left[\left(\Gamma_{k}, R_{k}, L_{k}, F_{k}\right) \in \mathcal{R}>0,1\right)\right]}, \\
& A^{>0,2}(\delta)=\sum_{k} R_{k}\left(\delta-\Gamma_{k}\right) 1_{\left.\left[\left(\Gamma_{k}, R_{k}, L_{k}, F_{k}\right) \in \mathcal{R}>0,2\right)\right]}, \\
& A^{<0,1}(\delta)=\sum_{k} R_{k}\left(L_{k}+\Gamma_{k}\right) 1_{\left.\left[\left(\Gamma_{k}, R_{k}, L_{k}, F_{k}\right) \in \mathcal{R}<0,1\right)\right]}, \\
& A^{<0,2}(\delta)=\sum_{k} R_{k} \delta 1_{\left.\left[\left(\Gamma_{k}, R_{k}, L_{k}, F_{k}\right) \in \mathcal{R}<0,2\right)\right]} .
\end{aligned}
$$

As a further notational device, we will adopt the convention that for a region $\mathcal{R}$ of the $(s, r, l, u)$-space, $A^{\mathcal{R}}\left(t_{1}, t_{2}\right]$ will denote the cumulative work inputted to the system in times $\left(t_{1}, t_{2}\right]$ from points $\left(\Gamma_{k}, R_{k}, L_{k}, F_{k}\right)$ in region $\mathcal{R}$.

We can represent the restrictions of $N$ to each of the 4 regions as given in (2.4) as empirical measures of a Poisson number of iid points whose joint distributions are the mean measure $\mu^{\#}$ restricted to that region and normalized to be a probability measure. (See, for instance, Resnick (1992, page 341).) For example

$$
N\left(\cdot \mathcal{R}^{>0,1}\right)=\sum_{k=1}^{P^{>0,1}(\delta)} \epsilon_{\left(\Gamma_{k}^{>0,1}, R_{k}^{>0,1}, L_{k}^{>0,1}, F_{k}^{>0,1}\right)}
$$


where $P^{>0,1}(\delta)$ is Poisson with parameter

$$
\begin{aligned}
\mu^{\#\left(\mathcal{R}^{>0,1}\right)} & =\int_{\mathcal{R}>0,1} \lambda d s P\left[\left(R_{1}, L_{1}, F_{1}\right) \in(d r, d l, d u)\right] \\
& =\int_{0}^{\delta} \lambda d s P\left[L_{1}+s<\delta\right]=\int_{0}^{\delta} \lambda F_{L}(\delta-s) d s=\delta \hat{F}_{L}(\delta),
\end{aligned}
$$

(where $\hat{F}_{L}(x)=\int_{0}^{x} F_{L}(y) d y$ ) and $\left\{\left(\Gamma_{k}^{>0,1}, R_{k}^{>0,1}, L_{k}^{>0,1}, F_{k}^{>0,1}\right)\right\}$ are iid with joint distribution

$$
\frac{\mu^{\#}\left(\cdot \cap \mathcal{R}^{>0,1}\right)}{\mu^{\#}\left(\mathcal{R}^{>0,1}\right)}
$$

In what follows, we sometimes use the convention that $P^{A}(\delta)$ is Poisson distributed with parameter equal to the mean measure of the region $A$.

2.1. Specifying dependence structure for $(R, L, F)$. Depending on the dependence structure of the triple $(R, L, F)$, it is possible to have different limit behavior for $\mathbf{A}(\delta)$ in (2.3). We distinguish two cases that we denote by RL and RF:

- RL - the r.v.s $R$ and $L$ are independent (cf, Maulik et al. (2002));

- RF - the r.v.s $R$ and $F$ are independent (see evidence of asymtptotic independence in Campos et al. (2005), Heffernan and Resnick (2005)).

We focus in this paper on the RF model. This choice has some statistical justification and seems natural since it assumes file size distributions are unaffected by network state. Even if transmission rates are functions of the network state, we would still get the required independence property of the RF model.

2.2. The model RF. In this model, as previously mentioned, we assume that the rates of transmissions are independent of the file sizes, and hence we have that the durations of transmission are computed by the following relation

$$
L=\frac{F}{R} .
$$

That means that the distribution tail of the random variable $L$ is given by (cf. Breiman's theorem in Breiman (1965))

$$
\bar{F}_{L}(l) \sim \mathbb{E}\left(\frac{1}{R}\right)^{\alpha_{F}} \bar{G}(l)
$$

provided we assume

$$
\mathbb{E}\left[\frac{1}{R}\right]^{\alpha_{F}+\eta}<\infty
$$

for some $\eta>0$.

Since our limiting procedure will shrink the observation window $(0, \delta]$, there is no hope to get a weak limit in (2.3) unless we increase the arrival rate $\lambda=\lambda(\delta)$ of sessions. Thus we adopt a heavy traffic limit theorem philosophy and imagine moving through a family of models indexed by $\delta$ as $\delta \downarrow 0$. A convenient and effective choice of $\lambda$ is defined in the following way

$$
\lambda(\delta)=\frac{1}{\delta \bar{F}_{R}\left(\delta^{-1}\right)} .
$$

Note that since $1<\alpha_{R}<2$, this choice of $\lambda$ guarantees that as $\delta \rightarrow 0$, both

$$
\lambda(\delta)=\frac{1}{\delta^{\alpha_{R}+1} L_{R}\left(\delta^{-1}\right)} \rightarrow \infty \quad \text { and } \quad \delta \lambda(\delta)=\frac{1}{\delta^{\alpha_{R}} L_{R}\left(\delta^{-1}\right)} \rightarrow \infty .
$$

Using assumption (2.7), the behavior of the rv's $A^{(\cdot)}(\delta)$ is as follows:

- $A^{<0,1}(\delta)$ is equal in distribution to $A^{>0,2}(\delta)$;

- $A^{<0,2}(\delta)$ does not converge weakly without scaling and with centering and scaling converges to a Gaussian rv; 
- $A^{>0,1}(\delta)$ converges in distribution to a compound poisson random variable;

- $A^{>0,2}(\delta)$, suitably centered, converges weakly to an infinitely divisible rv with finite variance and whose Lévy measure has a regularly varying tail with index $-\left(\alpha_{F}+\alpha_{R}\right)$, where $\alpha_{F}+\alpha_{R}>2$.

\section{Limits FOR CUMULATIVE INPUT $A(\delta)$}

We now present the details of the limiting arguments yielding distributional approximations for inputs from each of the four regions.

3.1. Region $\mathcal{R}^{>0,2}$. Recall this is the region contributing input in $(0, \delta]$ from sessions initiated in $(0, \delta]$ but terminating after $\delta$.

3.1.1. Characteristic function. For $\theta \in \mathbb{R}$, we compute

$$
\begin{aligned}
\mathbb{E}\left(e^{i \theta A^{>0,2}(\delta)}\right) & =\mathbb{E} \exp \left\{i \theta \sum_{i=1}^{P_{>0,2}(\delta)} R_{i}^{>0,2}\left(\delta-\Gamma_{i}^{>0,2}\right)\right\} \\
& =\exp \left\{\mathbb{E}\left(P^{>0,2}(\delta)\right)\left[\mathbb{E} e^{i \theta R_{1}^{>0,2}\left(\delta-\Gamma_{1}^{>0,2}\right)}-1\right]\right\} \\
& =\exp \left\{\iiint_{\substack { 0<s<\delta \\
\begin{subarray}{c}{r>l>\delta \\
r>0{ 0 < s < \delta \\
\begin{subarray} { c } { r > l > \delta \\
r > 0 } }\end{subarray}}\left(e^{i \theta r(\delta-s)}-1\right) \lambda d s F_{L, R}(d l, d r)\right\} \\
& =\exp \left\{\int_{0}^{\delta} \int_{r>0}\left(e^{i \theta r(\delta-s)}-1\right) P\left[\frac{F}{R}>\delta-s, R \in d r\right] \lambda d s\right\} \\
& =\exp \left\{\int_{s=0}^{\delta} \int_{r=0}^{\infty}\left(e^{i \theta r s}-1\right) \bar{G}(r s) F_{R}(d r) \lambda d s\right\} \\
& =\exp \left\{\lambda \int_{r=0}^{\infty} \int_{s=0}^{r \delta}\left(e^{i \theta s}-1\right) \bar{G}(s) r^{-1} F_{R}\left(\delta^{-1} d r\right) d s\right\} \\
& =\exp \left\{\int_{r=0}^{\infty}\left(\int_{s=0}^{r}\left(e^{i \theta s}-1\right) \bar{G}(s) d s\right) r^{-1} \frac{F_{R}\left(\delta^{-1} d r\right)}{\bar{F}_{R}\left(\delta^{-1}\right)}\right\}
\end{aligned}
$$

where we used the definition of $\lambda$ in (2.7). Interchanging the order of integration, we get

$$
=\exp \left\{\int_{s=0}^{\infty}\left(e^{i \theta s}-1\right) \bar{G}(s)\left(\int_{r=s}^{\infty} r^{-1} \mu_{\delta}(d r)\right) d s\right\}
$$

where

$$
\mu_{\delta}(d r):=\frac{F_{R}\left(\delta^{-1} d r\right)}{\bar{F}_{R}\left(\delta^{-1}\right)}
$$

Write

$$
\nu_{\delta}^{>0,2}(d s)=\left(\nu_{\delta}^{>0,2}\right)^{\prime}(s) d s=\bar{G}(s)\left(\int_{r=s}^{\infty} r^{-1} \mu_{\delta}(d r)\right) d s
$$

and we obtain

$$
\mathbb{E}\left(e^{i \theta A^{>0,2}(\delta)}\right)=\exp \left\{\int_{s=0}^{\infty}\left(e^{i \theta s}-1\right) \nu_{\delta}^{>0,2}(d s)\right\} .
$$


3.1.2. Properties of $\nu_{\delta}^{>0,2}$.

Proposition 1. As $\delta \rightarrow 0$,

$$
\nu_{\delta}^{>0,2} \stackrel{v}{\rightarrow} \nu_{0}^{>0,2}
$$

on $(0, \infty]$; that is, we have vague convergence to a limit. Further, the limit measure $\nu_{0}^{>0,2}$ is a Lévy measure with density

$$
\frac{\alpha_{R}}{1+\alpha_{R}} \bar{G}(x) x^{-\alpha_{R}-1}
$$

The tail of the Lévy measure is regularly varying with index $-\left(\alpha_{F}+\alpha_{R}\right)$.

Proof. Observe that for $s \geq 1$,

$$
\left(\nu_{\delta}^{>0,2}\right)^{\prime}(s)=\bar{G}(s) \int_{r=s}^{\infty} r^{-1} \mu_{\delta}(d r) \leq \bar{G}(s) \mu_{\delta}(s, \infty] s^{-1}
$$

and by Potter's bounds, for some small $\eta$, all $s \geq 1$, and for all sufficiently small $\delta$, we have the upper bound

$$
\leq c \bar{G}(s) s^{-\left(\alpha_{R}-\eta\right)-1}
$$

which is integrable with respect to Lebesgue measure on any neighborhood of $\infty$. Hence, by dominated convergence, for $x>0$,

$$
\begin{aligned}
\nu_{\delta}^{>0,2}(x, \infty] & =\int_{x}^{\infty}\left(\nu_{\delta}^{>0,2}\right)^{\prime}(s) d s \rightarrow \int_{x}^{\infty} \bar{G}(s)\left(\int_{s}^{\infty} r^{-1} \alpha_{R} r^{-\alpha_{R}-1} d r\right) d s \\
& =\nu_{0}^{>0,2}(x, \infty]=\frac{\alpha_{R}}{1+\alpha_{R}} \int_{x}^{\infty} \bar{G}(s) s^{-\alpha_{R}-1} d s .
\end{aligned}
$$

Regular variation of $\nu_{0}^{>0,2}(x, \infty]$ follows from the regular variation of the integrand in (3.3) and Karamata's theorem. In fact, as $x \rightarrow \infty$,

$$
\nu_{0}^{>0,2}(x, \infty] \sim \frac{\alpha_{R}}{\left(\alpha_{R}+1\right)\left(\alpha_{F}+\alpha_{R}\right)} x^{-\alpha_{R}} \bar{G}(x)
$$

To check $\nu_{0}^{>0,2}$ is a Lévy measure, note

$$
\int_{0}^{1} s^{2} \bar{G}(s) s^{-\alpha_{R}-1} d s \leq \int_{0}^{1} s^{2} s^{-\alpha_{R}-1} d s<\infty
$$

since $1<\alpha_{R}<2$.

3.1.3. Weak limit for $A^{>0,2}(\delta)$. Now we use (3.2) and write

(3.5) $\mathbb{E} \exp \left\{i \theta\left(A^{>0,2}(\delta)-\int_{0}^{1} s \nu_{\delta}^{>0,2}(d s)\right)\right\}=\exp \left\{\int_{1}^{\infty}\left(e^{i \theta s}-1\right) \nu_{\delta}^{>0,2}(d s)+\int_{0}^{1}\left(e^{i \theta s}-1-i \theta s\right) \nu_{\delta}^{>0,2}(d s)\right\}$.

The two integrals on the right in (3.5) each converge when $\delta \rightarrow 0$.

Proposition 2. As $\delta \rightarrow 0$ :

$$
\begin{aligned}
& \int_{1}^{\infty}\left(e^{i \theta s}-1\right) \nu_{\delta}^{>0,2}(d s) \rightarrow \int_{1}^{\infty}\left(e^{i \theta s}-1\right) \nu_{0}^{>0,2}(d s) \\
& \int_{0}^{1}\left(e^{i \theta s}-1-i \theta s\right) \nu_{\delta}^{>0,2}(d s) \rightarrow \int_{0}^{1}\left(e^{i \theta s}-1-i \theta s\right) \nu_{0}^{>0,2}(d s) .
\end{aligned}
$$

Therefore, as $\delta \rightarrow 0$

$$
A^{>0,2}(\delta)-\int_{0}^{1} s \nu_{\delta}^{>0,2}(d s) \Rightarrow X^{>0,2},
$$

where the limit random variable is infinitely divisible with Lévy measure $\nu_{0}^{>0,2}$ and characteristic function given by the right side of (3.5) with $\nu_{\delta}^{>0,2}$ replaced by $\nu_{0}^{>0,2}$. 
Proof. The convergence in (3.6) follows from standard weak convergence since the integrand is bounded and continuous and

weakly as probability measures on $(1, \infty]$.

$$
\frac{\nu_{\delta}^{>0,2}(\cdot)}{\nu_{\delta}^{>0,2}(1, \infty]} \Rightarrow \frac{\nu_{0}^{>0,2}(\cdot)}{\nu_{0}^{>0,2}(1, \infty]}
$$

For the proof of (3.7), observe that

$$
\begin{aligned}
\left|e^{i \theta s}-1-i \theta s\right|\left(\nu_{\delta}^{>0,2}\right)^{\prime}(s) & \leq \frac{\theta^{2} s^{2}}{2} \bar{G}(s) s^{-1} \mu_{\delta}(s, \infty] \\
& \leq c s \frac{\bar{F}_{R}\left(\delta^{-1} s\right)}{\bar{F}_{R}\left(\delta^{-1}\right)}=c \frac{V\left(\delta^{-1} s\right)}{V\left(\delta^{-1}\right)}
\end{aligned}
$$

where $V(s)=s \bar{F}_{R}(s)$ is regularly varying with index $-\alpha_{R}+1$. Now as $\delta \rightarrow 0$,

$$
\left|e^{i \theta s}-1-i \theta s\right|\left(\nu_{\delta}^{>0,2}\right)^{\prime}(s) \rightarrow\left|e^{i \theta s}-1-i \theta s\right|\left(\nu_{0}^{>0,2}\right)^{\prime}(s)
$$

and

Furthermore, by Karamata's theorem,

$$
\frac{V\left(\delta^{-1} s\right)}{V\left(\delta^{-1}\right)} \rightarrow s^{-\alpha_{R}+1}
$$

$$
\int_{0}^{1} \frac{V\left(\delta^{-1} s\right)}{V\left(\delta^{-1}\right)} d s \rightarrow \int_{0}^{1} s^{-\alpha_{R}+1} d s=\frac{1}{2-\alpha_{R}} .
$$

The desired result now follows from Pratt's lemma (Pratt (1960) or Resnick (1998, page 164)) since Pratt's lemma may be applied to both the real and imaginary parts of

$$
\left(e^{i \theta s}-1-i \theta s\right)\left(\nu_{\delta}^{>0,2}\right)^{\prime}(s)
$$

to get convergence to the limit after integrating on $[0,1]$.

3.2. Region $\mathcal{R}^{>0,1}$. Corresponding to this region, the traffic contribution is

$$
A^{>0,1}(\delta)=\sum_{i=1}^{P^{>0,1}(\delta)} F_{i}^{>0,1} .
$$

Now

$$
\begin{aligned}
\mathbb{E}\left(P^{>0,1}(\delta)\right) & =\iiint_{\substack{0<s<\delta \\
r>0 \\
0<s+u / r<\delta}} \lambda d s G(d u) F_{R}(d r) \\
& =\lambda \int_{s=0}^{\delta} P[F / R \leq s] d s=\lambda \int_{s=0}^{\delta} P\left[R / F \geq s^{-1}\right] d s \\
& =\lambda \int_{\delta^{-1}}^{\infty} P[R / F \geq s] \frac{d s}{s^{2}} .
\end{aligned}
$$

Applying Beiman's theorem (Breiman (1965)) after assuming that $\mathbb{E}\left(F^{-\left(\alpha_{R}+\eta\right)}\right)<\infty$ for some $\eta>0$ and as $\delta \rightarrow 0$, the above is asymptotic to

$$
\sim \lambda \int_{\delta^{-1}}^{\infty} P[R>s] \frac{d s}{s^{2}} \mathbb{E}\left(F^{-\alpha_{R}}\right)
$$

and applying Karamata's theorem, this is

$$
\begin{aligned}
& \sim \frac{\lambda \delta}{1+\alpha_{R}} P\left[R>\delta^{-1}\right] \mathbb{E}\left(F^{-\alpha_{R}}\right) \\
& \sim \frac{\mathbb{E}\left(F^{-\alpha_{R}}\right)}{1+\alpha_{R}} .
\end{aligned}
$$


Thus as $\delta \rightarrow 0$,

$$
\mathbb{E}\left(P^{>0,1}(\delta)\right) \rightarrow \frac{\mathbb{E}\left(F^{-\alpha_{R}}\right)}{1+\alpha_{R}} .
$$

This means

$$
P^{>0,1}(\delta) \Rightarrow P^{>0,1}(0)
$$

where $P^{>0,1}(0)$ is Poisson with parameter $\frac{\mathbb{E}\left(F^{-\alpha_{R}}\right)}{1+\alpha_{R}}$.

Now we observe that the distribution of $F_{1}^{>0,1}$ converges as $\delta \rightarrow 0$. We have for $x>0$,

$$
\begin{aligned}
\mathbb{E}\left(P^{>0,1}(\delta)\right) P\left[F_{1}^{>0,1} \leq x\right] & =\iint_{\substack{0<s<\delta \\
r>0 \\
s+u / r<\delta \\
u \leq x}} \lambda d s G(d u) F_{R}(d r) \\
& =\int_{s=0}^{\delta} P\left[F R^{-1} \leq s, F \leq x\right] \lambda d s \\
& =\lambda \int_{s=0}^{\delta} P\left[R F^{-1} \geq s^{-1}, F \leq x\right] d s \\
& =\lambda \int_{\delta^{-1}}^{\infty} P\left[R F^{-1} 1_{[F \leq x]} \geq s\right] \frac{d s}{s^{2}} \\
& \sim \frac{\lambda \delta}{1+\alpha_{R}} P\left[R>\delta^{-1}\right] \mathbb{E}\left(F^{-\alpha_{R}} 1_{[F \leq x]}\right) .
\end{aligned}
$$

We conclude, as $\delta \rightarrow 0$,

$$
P\left[F_{1}^{>0,1} \leq x\right] \Rightarrow \frac{\mathbb{E}\left(F^{-\alpha_{R}} 1_{[F \leq x]}\right)}{\mathbb{E}\left(F^{-\alpha_{R}}\right)} .
$$

This leads to the following result.

Proposition 3. Assume that

$$
\mathbb{E}(F)^{-\left(\alpha_{R}+\eta\right)}<\infty
$$

Then $A^{>0,1}(\delta)$ given in (3.8) is a compound Poisson random variable which, as $\delta \rightarrow 0$ converges weakly to a limiting compound Poisson random variable $X^{>0,1}$,

$$
X^{>0,1}=\sum_{i=1}^{P>0,1}(0)
$$

where $P^{>0,1}(0)$ is a Poisson random variable with parameter $\frac{\mathbb{E}\left(F^{-\alpha_{R}}\right)}{1+\alpha_{R}}$, independent of the iid sequence $\left\{R_{i}^{>0,1}(0), i \geq 1\right\}$ which has common distribution function

$$
\frac{\mathbb{E}\left(F^{-\alpha_{R}} 1_{[F \leq x]}\right)}{\mathbb{E}\left(F^{-\alpha_{R}}\right)} .
$$

The tail probabilities of this distribution, and hence of the limiting compound Poisson random variable, are regularly varying with index $-\left(\alpha_{R}+\alpha_{F}\right)$ and in fact, as $x \rightarrow \infty$,

$$
\frac{\mathbb{E}\left(F^{-\alpha_{R}} 1_{[F>x]}\right)}{\mathbb{E}\left(F^{-\alpha_{R}}\right)} \sim \frac{\alpha_{F}}{\alpha_{R}+\alpha_{F}} x^{-\alpha_{R}} \bar{G}(x) .
$$

3.3. The contribution of the region $\mathcal{R}^{<0,2}$. In the region $\mathcal{R}^{<0,2}$, we have contributions to traffic in $(0, \delta)$ from sessions starting prior to 0 and ending after $\delta$. 
3.3.1. Characteristic function of $A^{<0,2}(\delta)$. Since

$$
A^{<0,2}(\delta)=\sum_{i=1}^{P^{<0,2}(\delta)} R_{i}^{<0,2} \delta,
$$

the characteristic function of $A^{<0,2}(\delta)$ is computed as follows. For $\theta \in \mathbb{R}$,

$$
\begin{aligned}
\mathbb{E} e^{i \theta A^{<0,2}(\delta)} & =\exp \left\{\mathbb{E}\left(P^{<0,2}(\delta)\right)\left[\mathbb{E} e^{i \theta R_{1}^{<0,2} \delta}-1\right]\right\} \\
& =\exp \left\{\iiint_{\substack{s<0, r>0 \\
l>|s|+\delta}}\left(e^{i \theta r \delta}-1\right) \lambda d s F_{L, R}(d l, d r)\right\} \\
& =\exp \left\{\lambda \int_{s=\delta}^{\infty} \int_{r=0}^{\infty}\left(e^{i \theta r \delta}-1\right) \bar{G}(r s) d s F_{R}(d r)\right\}
\end{aligned}
$$

and reversing the order of integration and setting $\bar{G}_{0}(x)=\int_{x}^{\infty} \bar{G}(u) d u / \mathbb{E}(F)$ we get the above equal to

$$
\begin{aligned}
& =\exp \left\{\lambda \int_{r=0}^{\infty}\left(e^{i \theta r \delta}-1\right) r^{-1} \bar{G}_{0}(r \delta) \mathbb{E}(F) F_{R}(d r)\right\} \\
& =\exp \left\{\lambda \delta \int_{r=0}^{\infty}\left(e^{i \theta r}-1\right) r^{-1} \bar{G}_{0}(r) F_{R}\left(\delta^{-1} d r\right) \mathbb{E}(F)\right\} \\
& =\exp \left\{\int_{r=0}^{\infty}\left(e^{i \theta r}-1\right) r^{-1} \bar{G}_{0}(r) \frac{F_{R}\left(\delta^{-1} d r\right)}{\bar{F}\left(\delta^{-1}\right)} \mathbb{E}(F)\right\}
\end{aligned}
$$

Set, as before,

and define

$$
\mu_{\delta}(d r)=\frac{F_{R}\left(\delta^{-1} d r\right)}{\bar{F}_{R}\left(\delta^{-1}\right)}
$$

We conclude

$$
\nu_{\delta}^{<0,2}(d r)=\mathbb{E}(F) r^{-1} \bar{G}_{0}(r) \mu_{\delta}(d r)
$$

3.3.2. Properties of $\nu_{\delta}^{<0,2}$. The following properties of the measure $\nu_{\delta}^{<0,2}$ are evident.

(1) As $\delta \rightarrow 0$, the measures $\nu_{\delta}^{<0,2}$ converge vaguely on $(0, \infty]$ :

$$
\nu_{\delta}^{<0,2} \stackrel{v}{\rightarrow} \nu_{0}^{<0,2}
$$

where, for $x>0$

$$
\nu_{0}^{<0,2}(x, \infty]=\mathbb{E}(F) \int_{x}^{\infty} r^{-1} \bar{G}_{0}(r) \alpha_{R} r^{-\alpha_{R}-1} d r .
$$

(2) The tail of the measure, $\nu_{0}^{<0,2}(x, \infty]$ is regularly varying with index $-\left(\alpha_{R}+\alpha_{F}\right)$ and in fact,

$$
\begin{aligned}
\frac{\nu_{0}^{<0,2}(x, \infty]}{x^{-\alpha_{R}-1} \bar{G}_{0}(x)} & =\mathbb{E}(F) \int_{1}^{\infty} r^{-1} \frac{\bar{G}_{0}(x r)}{\bar{G}_{0}(x)} \alpha_{R} r^{-\alpha_{R}-1} d r \\
& \rightarrow \mathbb{E}(F) \int_{1}^{\infty} r^{-1} r^{-\left(\alpha_{F}-1\right)} \alpha_{R} r^{-\alpha_{R}-1} d r \\
& =\mathbb{E}(F) \frac{\alpha_{R}}{\alpha_{R}+\alpha_{F}} .
\end{aligned}
$$

(3) The measure $\nu_{0}^{<0,2}$ is not a Lévy measure since

$$
\int_{0}^{1} r^{2} \nu_{0}^{<0,2}(d r)=\alpha_{R} \mathbb{E}(F) \int_{0}^{1} r^{-\alpha_{R}} \bar{G}_{0}(r) d r
$$




$$
\geq \alpha_{R} \mathbb{E}(F) \bar{G}_{0}(1) \int_{0}^{1} r^{-\alpha_{R}} d r=\infty .
$$

This means we will not get an infinitely divisible weak limit without Gaussian component for $A^{<0,2}(\delta)$.

3.3.3. Gaussian limit. Observe that the quantity

$$
m(\delta):=\mathbb{E}(F) \int_{0}^{1} \bar{G}_{0}(r) \mu_{\delta}(d r)
$$

is finite, since

$$
m(\delta) \leq \mathbb{E}(F) \int_{0}^{1} 1 \mu_{\delta}(d r)=\mathbb{E}(F) \frac{F_{R}\left(\delta^{-1}\right)}{\bar{F}_{R}\left(\delta^{-1}\right)}<\infty .
$$

Also, define

$$
a(\delta):=\left(\mathbb{E}(F) \int_{0}^{1} r \bar{G}_{0}(r) \mu_{\delta}(d r)\right)^{1 / 2} .
$$

Note that as $\delta \rightarrow 0$, we have $a(\delta) \rightarrow \infty$ since for any $\eta>0$,

$$
\begin{aligned}
\liminf _{\delta \rightarrow 0} a^{2}(\delta) & \geq \liminf _{\delta \rightarrow 0} \mathbb{E}(F) \int_{\eta}^{1} r \bar{G}_{0}(r) \mu_{\delta}(d r)=\mathbb{E}(F) \int_{\eta}^{1} r \bar{G}_{0}(r) \alpha_{R} r^{-\alpha_{R}-1} d r \\
& \geq\left.\frac{\mathbb{E}(F)}{1-\alpha_{R}} \bar{G}_{0}(1) r^{-\alpha_{R}+1}\right|_{\eta} ^{1} \rightarrow \infty,
\end{aligned}
$$

as $\eta \downarrow 0$.

Now we use (3.12) and write

$$
\begin{aligned}
\mathbb{E} \exp \left\{i \theta\left[A^{<0,2}(\delta)-m(\delta)\right] / a(\delta)\right\} \\
=\exp \left\{\int_{0}^{\infty}\left(e^{i a^{-1}(\delta) \theta r}-1\right) \nu_{\delta}^{<0,2}(d r)-i \theta \frac{\mathbb{E}(F)}{a(\delta)} \int_{0}^{1} \bar{G}_{0}(r) \mu_{\delta}(d r)\right\} \\
=\exp \left\{\int_{0}^{1}\left(e^{i a^{-1}(\delta) \theta r}-1-i \frac{\theta}{a(\delta)} r\right) \mathbb{E}(F) r^{-1} \bar{G}_{0}(r) \mu_{\delta}(d r)+\int_{1}^{\infty}\left(e^{i a^{-1}(\delta) \theta r}-1\right) \nu_{\delta}^{<0,2}(d r)\right\} \\
=\exp \{A+B\} .
\end{aligned}
$$

Now

$$
\begin{aligned}
|B| & \leq \mathbb{E}(F) \int_{1}^{\infty}|\theta| \frac{r}{a(\delta)} r^{-1} \bar{G}_{0}(r) \mu_{\delta}(d r) \\
& \leq O\left(\frac{1}{a(\delta)}\right) \rightarrow 0,
\end{aligned}
$$

as $\delta \rightarrow 0$, since $a(\delta) \rightarrow \infty$. For $A$ we have $A \rightarrow-\theta^{2} / 2$ since

$$
\begin{aligned}
\mid \int_{0}^{1}\left(e^{i \theta a^{-1}(\delta) r}-\right. & \left.1-i \frac{\theta}{a(\delta)} r\right) \mathbb{E}(F) r^{-1} \bar{G}_{0}(r) \mu_{\delta}(d r)+\frac{\theta^{2}}{2} \mid \\
= & \left|\int_{0}^{1}\left(e^{i \theta a^{-1}(\delta) r}-1-i \frac{\theta}{a(\delta)} r-\frac{1}{2}\left(\frac{i \theta r}{a(\delta)}\right)^{2}\right) \mathbb{E}(F) r^{-1} \bar{G}_{0}(r) \mu_{\delta}(d r)\right| \\
\leq & \frac{1}{a^{3}(\delta)} \int_{0}^{1} \frac{1}{3 !}|\theta|^{3} r^{3} \mathbb{E}(F) r^{-1} \bar{G}_{0}(r) \mu_{\delta}(d r) .
\end{aligned}
$$

For any $\eta>0$, split the integral

$$
\int_{0}^{1}=\int_{0}^{\eta}+\int_{\eta}^{1}
$$


and

$$
\begin{aligned}
\frac{1}{a^{3}(\delta)} \int_{0}^{1} r^{2} \bar{G}_{0}(r) \mu_{\delta}(d r) & \sim \frac{1}{a^{3}(\delta)} \int_{0}^{\eta} r^{2} \bar{G}_{0}(r) \mu_{\delta}(d r) \\
& \leq \frac{\eta}{a(\delta)^{3}} \int_{0}^{\eta} r \bar{G}_{0}(r) \mu_{\delta}(d r) \\
& \sim \frac{\eta}{a(\delta)^{3}} \int_{0}^{1} r \bar{G}_{0}(r) \mu_{\delta}(d r) \\
& =O\left(\eta \cdot \frac{1}{a(\delta)}\right) \rightarrow 0 .
\end{aligned}
$$

We summarize.

Proposition 4. With $m(\delta)$ defined by (3.13) and a $(\delta)$ given by (3.14), we have

$$
\frac{A^{<0,2}(\delta)-m(\delta)}{a(\delta)} \Rightarrow X^{<0,2} \sim N(0,1)
$$

as $\delta \rightarrow 0$.

Remark 1. The centering may be changed from $m(\delta)$ to

$$
m^{\#}(\delta):=\mathbb{E}\left(\sum_{i=1}^{P^{<0,2}(\delta)} R_{i}^{<0,2} \delta\right)=\mathbb{E}\left(P^{<0,2}(\delta)\right) \mathbb{E}\left(R^{<0,2} \delta\right)
$$

since

$$
\begin{aligned}
\mathbb{E}\left(P^{<0,2}(\delta)\right) \mathbb{E}\left(R^{<0,2} \delta\right) & =\iiint_{\substack{s<0, r>0 \\
l>\delta+|s|}} r \delta \lambda d s F_{L}(d l, d r) \\
& =\int_{s=\delta}^{\infty} \int_{r=0}^{\infty} r \delta \bar{G}(r s) F_{R}(d r) \lambda d s \\
& =\lambda \delta \int_{r=0}^{\infty} \bar{G}_{0}(r \delta) F_{R}(d r) \mathbb{E}(F) \\
& =\int_{r=0}^{\infty} \bar{G}_{0}(r) \frac{F_{R}\left(\delta^{-1} d r\right)}{\bar{F}_{R}\left(\delta^{-1}\right)} \mathbb{E}(F) \\
& =m(\delta)+\mathbb{E}(F) \int_{1}^{\infty} \bar{G}_{0}(r) \mu_{\delta}(d r) \\
& =m(\delta)+o(a(\delta)) .
\end{aligned}
$$

Similarly, the scaling $a(\delta)$ may be changed to

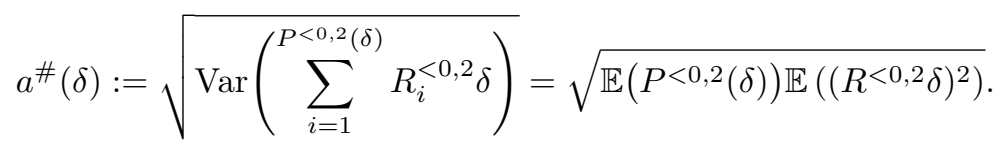

This follows from

$$
\begin{aligned}
\mathbb{E}\left(P^{<0,2}(\delta)\right) \mathbb{E}\left(\left(R^{<0,2} \delta\right)^{2}\right) & =\iiint_{\substack{s<0, r>0 \\
l>|s|+\delta}} \delta^{2} r^{2} \lambda d s F_{L, R}(d l, d r) \\
& =\int_{s=\delta}^{\infty} \int_{r>0} \delta^{2} r^{2} \lambda d s \bar{G}(r s) F_{R}(d r) \\
& =\lambda \int_{r=0}^{\infty} \delta^{2} r^{2} \int_{s=\delta}^{\infty} \bar{G}(r s) d s F_{R}(d r) \\
& =\lambda \delta \int_{r=0}^{\infty} \delta r \int_{s=r \delta}^{\infty} \bar{G}(s) d s F_{R}(d r)
\end{aligned}
$$




$$
\begin{aligned}
& =\lambda \delta \int_{r=0}^{\infty} r \bar{G}_{0}(r) F_{R}\left(\delta^{-1} d r\right) \mathbb{E}(F) \\
& =\int_{r=0}^{\infty} r \bar{G}_{0}(r) \mu_{\delta}(d r) \mathbb{E}(F) .
\end{aligned}
$$

Note, as $\delta \rightarrow 0$,

since $1<\alpha_{R}<2$. Therefore

$$
\int_{1}^{\infty} r \bar{G}_{0}(r) \mu_{\delta}(d r) \rightarrow \int_{1}^{\infty} r \bar{G}_{0}(r) \alpha_{R} r^{-\alpha_{R}-1} d r<\infty
$$

$$
a^{2}(\delta) \sim \int_{0}^{\infty} r \bar{G}_{0}(r) \mu_{\delta}(d r) \mathbb{E}(F),
$$

as claimed.

3.3.4. Further properties of scaling $a(\delta)$. The scaling function plays a significant role in understanding dependence across time slots. Here are two properties we need in the next section.

Proposition 5. (a) For any $t>0$,

$$
\lim _{\delta \rightarrow 0} \frac{\int_{0}^{1} r \bar{G}_{0}(t r) \mu_{\delta}(d r)}{\int_{0}^{1} r \mu_{\delta}(d r)}=1 .
$$

(b) The growth rate of $a(\delta)$ is given by

$$
a^{2}(\delta)=\mathbb{E}(F) \int_{0}^{1} r \bar{G}_{0}(r) \mu_{\delta}(d r) \sim \mathbb{E}(F) \int_{0}^{1} r \mu_{\delta}(d r) \sim \mathbb{E}(F) \mathbb{E}(R) \frac{\left(\delta^{-1}\right)^{\left(\alpha_{R}-1\right)}}{L_{R}\left(\delta^{-1}\right)} .
$$

Proof. (a) Since $\bar{G}_{0} \leq 1$, we see 1 is an upperbound of the ratio in (3.18). To get a lower bound, observe that $\int_{0}^{1} r \mu_{\delta}(d r) \rightarrow \infty$ as $\delta \rightarrow 0$, since for any $\eta>0$,

since $1<\alpha_{R}<2$. Therefore,

$$
\begin{aligned}
\int_{0}^{1} r \mu_{\delta}(d r) & \geq \int_{\eta}^{1} r \mu_{\delta}(d r) \rightarrow \int_{\eta}^{1} r \alpha_{R} r^{-\alpha_{R}-1} d r \\
& =\frac{\alpha_{R}}{\alpha_{R}-1}\left[\eta^{-\left(\alpha_{R}-1\right)}-1\right] \rightarrow \infty \quad(\eta \downarrow 0),
\end{aligned}
$$

$$
\begin{aligned}
\frac{\int_{0}^{1} r \bar{G}_{0}(t r) \mu_{\delta}(d r)}{\int_{0}^{1} r \mu_{\delta}(d r)} & \geq \frac{\int_{0}^{\eta} r \bar{G}_{0}(t r) \mu_{\delta}(d r)}{\int_{0}^{1} r \mu_{\delta}(d r)} \geq \frac{\bar{G}_{0}(t \eta) \int_{0}^{\eta} r \mu_{\delta}(d r)}{\int_{0}^{1} r \mu_{\delta}(d r)} \\
& =\bar{G}_{0}(t \eta) \frac{\int_{0}^{1} r \mu_{\delta}(d r)}{\int_{0}^{1} r \mu_{\delta}(d r)}+o(1) \\
& \rightarrow \bar{G}_{0}(t \eta) \quad(\delta \rightarrow 0) \\
& \rightarrow 1 \quad(\eta \rightarrow 0) .
\end{aligned}
$$

(b) To see the growth rate of $a(\delta)$, observe that

$$
\begin{aligned}
\int_{0}^{1} r \mu_{\delta}(d r) & =\int_{r=0}^{1}\left(\int_{v=0}^{r} d v\right) \mu_{\delta}(d r)=\int_{v=0}^{1} \int_{r=v}^{1} \mu_{\delta}(d r) d v \\
& =\int_{0}^{1} \mu_{\delta}(v, \infty] d v-\mu_{\delta}(1, \infty]=\frac{\int_{0}^{\delta^{-1}} \bar{F}_{R}(v) d v}{\delta^{-1} \bar{F}_{R}\left(\delta^{-1}\right)}-1 .
\end{aligned}
$$

We conclude

$$
\delta^{-1} \bar{F}_{R}\left(\delta^{-1}\right)\left[1+\int_{0}^{1} r \mu_{\delta}(d r)\right] \rightarrow \mathbb{E}(R) .
$$

This coupled with $\int_{0}^{1} r \mu_{\delta}(d r) \rightarrow \infty$ proves the result. 
3.4. Contribution of the region $\mathcal{R}^{<0,1}$. In this section, we prove that

$$
A^{<0,1}(\delta) \stackrel{d}{=} A^{>0,2}(\delta) .
$$

The idea behind this is as follows. Recall

$$
A^{<0,1}(\delta)=\sum_{k} R_{k}\left(L_{k}+\Gamma_{k}\right) 1_{\left[\left(\Gamma_{k}, R_{k}, L_{k}, F_{k}\right) \in \mathcal{R}<0,1\right)} .
$$

It is well known that in the $M / G / \infty$ model, the departure process has the same distribution as the arrival process, namely it is Poisson with rate $\lambda$. The process $A^{<0,1}(\delta)$ accumulates the contribution from 0 to $\delta$ of those sessions ending in $(0, \delta)$ and starting before time 0 . However, we may reindex Poisson points by swapping the termination and starting times. Now the region $A^{<0,1}(\delta)$ will correspond to the sessions starting in $(0, \delta]$ and terminating outside it which is exactly the contribution of the region $\mathcal{R}^{>0,2}$, namely $A^{>0,2}(\delta)$. A more formal proof is given in the following proposition.

Proposition 6. We have

$$
A^{<0,1}(\delta) \stackrel{d}{=} A^{>0,2}(\delta)
$$

and therefore, as $\delta \rightarrow 0$,

$$
A^{<0,1}(\delta)-\int_{0}^{1} s \nu_{\delta}^{<0,1}(d s) \Rightarrow X^{<0,1}
$$

where $\nu_{\delta}^{<0,1}=\nu_{\delta}^{>0,2}$ and $X^{<0,1} \stackrel{d}{=} X^{>0,2}$ with the quantities indexed by ${ }^{>0,2}$ defined as in Proposition 3.

Proof. We compute the characteristic function as follows. For $\theta \in \mathbb{R}$,

$$
\begin{aligned}
& \mathbb{E}\left(\exp \left\{i \theta \sum_{i=1}^{P^{<0,1}(\delta)} R_{i}^{<0,1}\left(\Gamma_{i}^{<0,1}+L_{i}^{<0,1}\right)\right\}\right)=\exp \left\{\mathbb{E}\left(P^{<0,1}(\delta)\right)\left[\mathbb{E}\left(e^{i \theta R_{1}^{<0,1}\left(\Gamma_{1}^{<0,1}+L_{1}^{<0,1}\right)}\right)-1\right]\right\} \\
&=\exp \left\{\iiint_{s<0, r>0}\left(e^{i \theta r(s+l)}-1\right) \lambda d s F_{L, R}(d l, d r)\right\} \\
&=\exp \left\{\int_{s=0}^{\infty} \int_{r>0}^{\infty} \int_{s<l \leq s+\delta}\left(e^{i \theta r(l-s)}-1\right) \lambda d s F_{R, L}(d r, d l)\right\} \\
&=\exp \left\{\lambda \int_{r=0}^{\infty} \int_{s=0}^{\infty} \int_{r s<u \leq r(s+\delta)}^{\infty}\left(e^{i \theta(u-r s)}-1\right) d s G(d u) F_{R}(d r)\right\} \\
&=\exp \left\{\lambda \int_{r=0}^{\infty} \int_{s=0}^{\infty} \int_{s<u \leq(s+r \delta)}\left(e^{i \theta(u-s)}-1\right) d s G(d u) r^{-1} F_{R}(d r)\right\} \\
&=\exp \left\{\lambda \int_{r=0}^{\infty} \int_{u=0}^{\infty} \int_{s \in[(u-r \delta) \vee 0, u]}\left(e^{i \theta(u-s)}-1\right) d s G(d u) r^{-1} F_{R}(d r)\right\} \\
&=\exp \left\{\lambda \int _ { r = 0 } ^ { \infty } \left(\int_{u=0}^{r \delta} \int_{s=0}^{u}\left(e^{i \theta(u-s)}-1\right) d s G(d u)\right.\right. \\
&\left.\left.+\int_{u=r \delta}^{\infty} \int_{s=u-r \delta}^{u}\left(e^{i \theta(u-s)}-1\right) d s G(d u)\right) r^{-1} F_{R}(d r)\right\} \\
&=\exp \left\{\lambda \int _ { r = 0 } ^ { \infty } \left(\int_{u=0}^{r \delta} \int_{s=0}^{u}\left(e^{i \theta s}-1\right) d s G(d u)\right.\right. \\
&\left.\left.+\int_{u=r \delta}^{\infty} \int_{s=0}^{r \delta}\left(e^{i \theta s}-1\right) d s G(d u)\right) r^{-1} F_{R}(d r)\right\}
\end{aligned}
$$


and reversing the order of integration for $u$ and $s$ yields

$$
\begin{aligned}
& =\exp \left\{\lambda \int_{r=0}^{\infty} \int_{s=0}^{r \delta}\left(e^{i \theta s}-1\right) \bar{G}(s) d s r^{-1} F_{R}(d r)\right\} \\
& =\exp \left\{\lambda \int_{s=0}^{\infty} \int_{r>s / \delta}\left(e^{i \theta s}-1\right) \bar{G}(s) d s r^{-1} F_{R}(d r)\right\} \\
& =\exp \left\{\lambda \delta \int_{s=0}^{\infty}\left(e^{i \theta s}-1\right) \bar{G}(s) \int_{r>s} r^{-1} F_{R}\left(\delta^{-1} d r\right) d s\right\} \\
& =\exp \left\{\int_{s=0}^{\infty}\left(e^{i \theta s}-1\right) \nu_{\delta}^{>0,2}(d s)\right\}
\end{aligned}
$$

where we used the notation of (3.1). This is the same characteristic function we found for $A^{>0,2}(\delta)$ in (3.2).

3.5. Discussion and summary. We summarize the contributions of the four regions to cumulative traffic in $(0, \delta)$.

(1) For the region $\mathcal{R}^{>0,2}$, we have, as $\delta \rightarrow 0$,

$$
X^{>0,2}(\delta):=A^{>0,2}(\delta)-\int_{0}^{1} s \nu_{\delta}^{>0,2}(d s) \Rightarrow X^{>0,2},
$$

a spectrally positive, infinitely divisible random variable with Lévy measure $\nu_{0}^{>0,2}$ with tail probabilities which are regularly varying with index $-\left(\alpha_{F}+\alpha_{R}\right)$. Observe $\alpha_{F}+\alpha_{R}>2$ so $\mathbb{E}\left(X^{>0,2}\right)^{2}<\infty$.

(2) For the region $\mathcal{R}^{>0,1}$, we have

$$
A^{>0,2}(\delta) \Rightarrow X^{>0,1}
$$

a compound Poisson random variable with tail probabilities which are regularly varying with index $-\left(\alpha_{F}+\alpha_{R}\right)$. Note

$$
P\left[X^{>0,1}>x\right] \sim c P\left[X^{>0,2}>x\right], \quad(x \rightarrow \infty) .
$$

(3) For the region $\mathcal{R}^{<0,2}$, we have

$$
X^{<0,2}(\delta):=\frac{A^{<0,2}(\delta)-m(\delta)}{a(\delta)} \Rightarrow X^{<0,2} \sim N(0,1) .
$$

(4) For the region $\mathcal{R}^{>0,2}$, we have

$$
A^{<0,1}(\delta) \stackrel{d}{=} A^{>0,2}(\delta)
$$

so

$$
X^{<0,1}(\delta):=A^{<0,1}(\delta)-\int_{0}^{1} s \nu_{\delta}^{>0,2}(d s) \Rightarrow X^{<0,1} \stackrel{d}{=} X^{>0,2} .
$$

This means we may write

$$
A(\delta)=X^{>0,2}(\delta)+\int_{0}^{1} s \nu_{\delta}^{>0,2}(d s)+A^{>0,1}(\delta)+a(\delta) X^{<0,2}(\delta)+m(\delta)+X^{<0,1}(\delta)+\int_{0}^{1} s \nu_{\delta}^{>0,2}(d s) .
$$

We conclude

$$
A(\delta)-m(\delta)-2 \int_{0}^{1} s \nu_{\delta}^{>0,2}(d s)=X^{>0,2}(\delta)+A^{>0,1}(\delta)+a(\delta) X^{<0,2}(\delta),
$$

where the summands on the right are independent and

$$
\begin{aligned}
& X^{<0,1}(\delta) \stackrel{d}{=} X^{>0,2}(\delta) \Rightarrow X^{>0,2} \quad \text { (infinitely divisible) } \\
& A^{>0,1}(\delta) \Rightarrow X^{>0,1}, \quad \text { (compound Poisson) } \\
& X^{<0,2}(\delta) \Rightarrow X^{<0,2} \quad \text { (normal). }
\end{aligned}
$$


Also,

$$
\frac{A(\delta)-m(\delta)-2 \int_{0}^{1} s \nu_{\delta}^{>0,2}(d s)}{a(\delta)} \Rightarrow X^{<0,2} \sim N(0,1) .
$$

Looking at the decomposition (3.22) and also at (3.23), the centered cumulative traffic inputted in the time slot $(0, \delta]$ has an asymptotically normal component on spatial scale $a(\delta)$ plus a component which asymptotically mixes an infinitely divisible and compound Poisson distribution. For traffic at fine time scales with high degree of aggregation resulting in a large number of sessions, the Gaussian component will thus obscure the more spikey, high rate transmissions represented in our model by the infinitely divisible and compound Poisson components.

This helps to explain why measurements with very high traffic aggregation report a Gaussian distribution. The normal component is due to the sessions that start before the time slot and end after the time slot. So they will be also responsible for the dependence structure of the process. As we see in the next section, the infinitely divisible and compound Poisson components contribute to the dependence across time slots in a manner which is of lower order than the Gaussian components.

\section{Dependence structure across slots.}

We now analyze the weak limits of the stochastic process

$$
\mathbf{A}(\delta):=\{A(k \delta,(k+1) \delta],-\infty<k<\infty\}
$$

defined in (2.3). We will see that the $\mathbb{R}^{\infty}$ family indexed by $\delta$ converges to a limiting Gaussian sequence

$$
\boldsymbol{X}_{\infty}=\left(X_{\infty}(k),-\infty<k<\infty\right\}
$$

with

$$
\operatorname{Corr}\left(X_{\infty}(0), X_{\infty}(k)\right)=1 .
$$

The price paid for letting $\delta \rightarrow 0$ is thus a limit sequence with degenerate dependence structure. The consequence of sampling at too high frequency (using economic terminology) is perfect correlation. However, we will see that for fixed $\delta>0$, we have long range dependence across time slots.

We begin by considering convergence of finite dimensional distributions.

4.1. Convergence of finite dimensional distributions. In this section we prove the following result.

Proposition 7. For any non-negative integer $k$, as $\delta \rightarrow 0$ we have in $\mathbb{R}^{k+1}$,

$$
\frac{1}{a(\delta)}\left[\left(\begin{array}{c}
A(0, \delta] \\
A(\delta, 2 \delta] \\
\vdots \\
A(k \delta,(k+1) \delta]
\end{array}\right)-\left\{2 \int_{0}^{1} v \bar{G}(v) \int_{r=v}^{\infty} r^{-1} \mu_{\delta}(d r) d v-\int_{0}^{1} \mathbb{E}(F) \bar{G}_{0}(r, \infty] \mu_{\delta}(d r)\right\}\left(\begin{array}{c}
1 \\
1 \\
\vdots \\
1
\end{array}\right)\right] \Rightarrow\left(\begin{array}{c}
X_{\infty}(0) \\
X_{\infty}(1) \\
\vdots \\
X_{\infty}(k)
\end{array}\right)
$$

where $X_{\infty}(i) \sim N(0,1)$ for $0 \leq i \leq k$ and

$$
\operatorname{Corr}\left(X_{\infty}(i), X_{\infty}(j)\right)=1 .
$$

Proof. Along with the regions $\mathcal{R}^{<0,1}, \mathcal{R}^{<0,2}, \mathcal{R}^{>0,1}, \mathcal{R}^{>0,2}$ used to analyze the convergence in distribution of $A(0, \delta]$, we need the analogously defined regions $\mathcal{R}^{<k \delta, 1}, \mathcal{R}^{<k \delta, 2}, \mathcal{R}^{>k \delta, 1}, \mathcal{R}^{>k \delta, 2}$, where for example

$$
\begin{aligned}
& \mathcal{R}^{<k \delta, 2}=\{(s, r, l, u): s<k \delta, s+l>(k+1) \delta\} \\
& \mathcal{R}^{>k \delta, 2}=\{(s, r, l, u): k \delta<s<(k+1) \delta, s+l>(k+1) \delta\} .
\end{aligned}
$$

See Figure 2. 


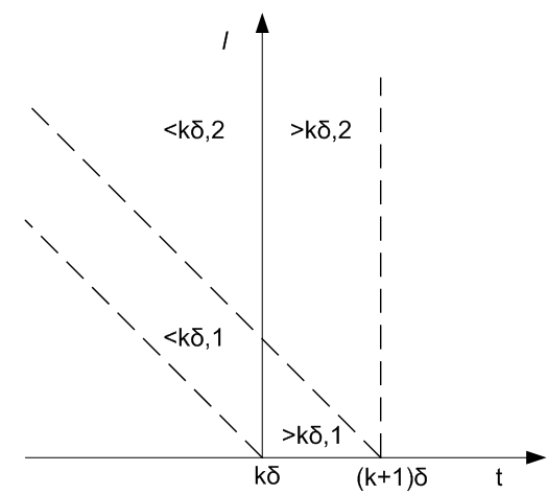

Figure 2. Four regions for analyzing contributions in $k$-th slot.

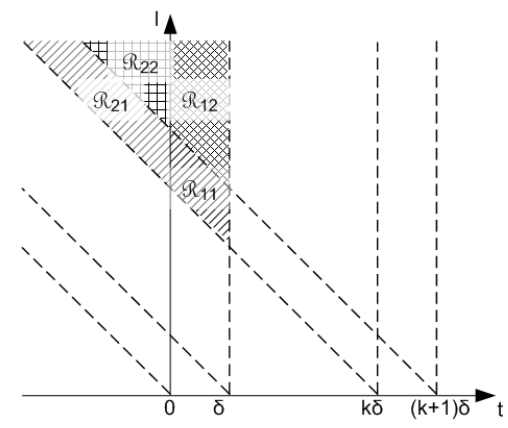

FiguRE 3. Regions for dependence analysis.

Additionally, for analyzing dependence between $A(0, \delta]$ and $A(k \delta,(k+1) \delta]$, we will need the regions $\mathcal{R}_{11}, \mathcal{R}_{12}, \mathcal{R}_{21}, \mathcal{R}_{22}$ which contain points $\left(\Gamma_{k}, R_{k}, L_{k}, F_{k}\right)$ contributing to both $A(0, \delta]$ as well as $A(k \delta,(k+1) \delta]$. (See Figure 3.) In particular, points in $\mathcal{R}_{22}=\mathcal{R}^{<0,2} \cap \mathcal{R}^{<k \delta, 2}$ contribute

$$
A^{\mathcal{R}_{22}}=\sum_{k:\left(\Gamma_{k}, R_{k}, L_{k}, F_{k}\right) \in \mathcal{R}_{22}} R_{k} \delta
$$

to both $A(0, \delta]$ and $A(k \delta,(k+1) \delta]$.

4.1.1. Behavior of $A^{\mathcal{R}_{22}}$. By analogy with (3.11), $A^{\mathcal{R}_{22}}$ is a Poissonized sum of iid rv's and we compute its characteristic function in a similar manner to achieve

$$
\mathbb{E}\left(e^{i \theta A^{\mathcal{R}_{22}}}\right)=\exp \left\{\iiint_{\substack{s<0, r>0 \\ l>(k+1) \delta+|s|}}\left(e^{i \theta r \delta}-1\right) \lambda d s F_{L, R}(d l, d r)\right\}
$$

and repeating the calculation which led to (3.12), we get this equal to

$$
=\exp \left\{\int_{0}^{\infty}\left(e^{i \theta r}-1\right) \mathbb{E}(F) \bar{G}_{0}((k+1) r) r^{-1} \mu_{\delta}(d r) .\right\}
$$

Setting

$$
\begin{aligned}
a_{k}^{2}(\delta) & =: \int_{0}^{1} \mathbb{E}(F) \bar{G}_{0}((k+1) r) r \mu_{\delta}(d r) \\
m_{k}(\delta) & =: \int_{0}^{1} \mathbb{E}(F) \bar{G}_{0}((k+1) r) \mu_{\delta}(d r)
\end{aligned}
$$




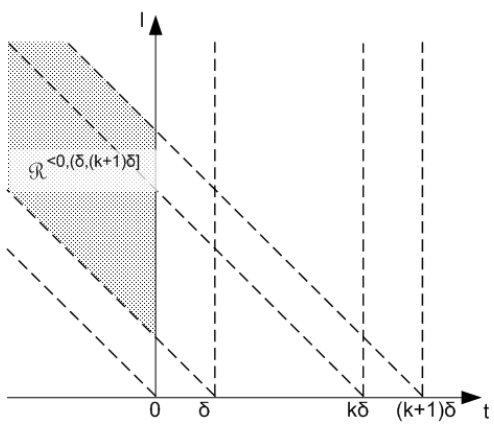

FiguRE 4. Region $\mathcal{R}^{<0,(\delta,(k+1) \delta]}$ for dependence analysis.

we find

$$
\frac{A^{\mathcal{R}_{22}}-m_{k}(\delta)}{a_{k}(\delta)} \Rightarrow X^{\mathcal{R}_{22}} \sim N(0,1)
$$

in $\mathbb{R}$, as $\delta \rightarrow 0$.

Note from Proposition 5, we have, as $\delta \rightarrow 0$,

$$
a_{k}(\delta) \sim a(\delta) \sim\left(\mathbb{E}(F) \int_{0}^{1} r \mu_{\delta}(d r)\right)^{1 / 2} .
$$

4.1.2. Contributions from other regions. Set

$$
\mathcal{R}^{<0,(\delta,(k+1) \delta]}=\{(s, r, l, u): s<0, \delta<|s|+l \leq(k+1) \delta\}
$$

(see Figure 4) and write

$$
\begin{aligned}
A(0, \delta] & =A^{>0,1}(0, \delta]+A^{>0,2}(0, \delta]+A^{<0,1}(0, \delta]+A^{<0,2}(0, \delta] \\
& =A^{>0,1}(0, \delta]+A^{>0,2}(0, \delta]+\left[A^{<0,1}(0, \delta]+A^{\mathcal{R}^{<0,(\delta,(k+1) \delta]}}(0, \delta]\right]+A^{R_{22}}(0, \delta] .
\end{aligned}
$$

Now mimicking the calculation in Section 3.3.1, we find

$$
\mathbb{E}\left(e^{i \theta A^{<0,(\delta,(k+1) \delta]}(0, \delta]}\right)=\exp \left\{\int_{0}^{\infty}\left(e^{i \theta r}-1\right) \mathbb{E}(F) r^{-1} G_{0}(r,(k+1) r] \mu_{\delta}(d r)\right\}
$$

and

$$
\nu_{\delta}^{<0,(\delta,(k+1) \delta]}(d r):=\mathbb{E}(F) r^{-1} G_{0}(r,(k+1) r] \mu_{\delta}(d r)
$$

converges to a Lévy measure with density

$$
\mathbb{E}(F) r^{-1} G_{0}(r,(k+1) r] \alpha_{R} r^{-\alpha_{R}-1} d r .
$$

This means that

$$
A^{\mathcal{R}^{<0,(\delta,(k+1) \delta]}}(0, \delta]-\int_{0}^{1} \mathbb{E}(F) G_{0}(r,(k+1) r] \mu_{\delta}(d r)
$$

converges to an infinitely divisible random variable with Lévy measure whose density is given by (4.8) and hence $\left.A^{\mathcal{R}^{<0,(\delta,(k+1) \delta]}}(0, \delta]-\int_{0}^{1} \mathbb{E}(F) G_{0}(r,(k+1) r] \mu_{\delta}\right)$ is $o_{p}(a(\delta))$.

We conclude that

$$
\begin{gathered}
A(0, \delta]-2 \int_{0}^{1} v \bar{G}(v) \int_{r=v}^{\infty} r^{-1} \mu_{\delta}(d r) d v-\int_{0}^{1} \mathbb{E}(F) G_{0}(r,(k+1) r] \mu_{\delta}(d r)-\int_{0}^{1} \mathbb{E}(F) \bar{G}_{0}((k+1) r) \mu_{\delta}(d r) \\
=A(0, \delta]-2 \int_{0}^{1} v \bar{G}(v) \int_{r=v}^{\infty} r^{-1} \mu_{\delta}(d r) d v-\int_{0}^{1} \mathbb{E}(F) \bar{G}_{0}(r, \infty] \mu_{\delta}(d r) \\
=A^{\mathcal{R}_{22}}(0, \delta]-m_{k}(\delta)+o_{p}(a(\delta)) .
\end{gathered}
$$




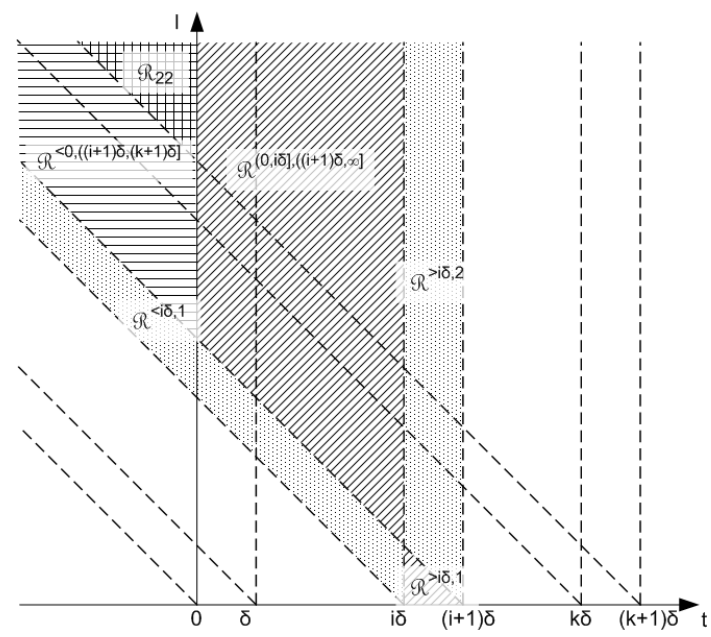

FiguRE 5. Regions for dependence analysis.

Likewise, we consider $A(i \delta,(i+1) \delta]$ for $1 \leq i \leq k$. We set

$$
\begin{aligned}
\mathcal{R}^{<0,((i+1) \delta,(k+1) \delta]} & =\{(s, r, l, u): s<0,(i+1) \delta<s+l<(k+1) \delta\} \\
\mathcal{R}^{(0, i \delta],((i+1) \delta, \infty]} & =\{(s, r, l, u): 0<s \leq i \delta ; s+l>(i+1) \delta\}
\end{aligned}
$$

(see Figure 5) and write

$$
\begin{aligned}
A(i \delta,(i+1) \delta]=A^{\mathcal{R}^{>i \delta, 1}}(i \delta & ,(i+1) \delta]+A^{\mathcal{R}^{>i \delta, 2}}(i \delta,(i+1) \delta]+A^{\mathcal{R}^{<i \delta, 1}}(i \delta,(i+1) \delta] \\
& +\left[A^{\mathcal{R}^{<0,((i+1) \delta,(k+1) \delta]}}(i \delta,(i+1) \delta]+A^{\mathcal{R}_{22}}(i \delta,(i+1) \delta]+A^{\left.\mathcal{R}^{(0, i \delta],((i+1) \delta, \infty]}(i \delta,(i+1) \delta]\right]}\right.
\end{aligned}
$$

Now

$$
\begin{aligned}
\mathbb{E} e^{i \theta A^{\mathcal{R}<0,((i+1) \delta,(k+1) \delta]}(i \delta,(i+1) \delta]} & =\exp \left\{\int_{0}^{\infty}\left(e^{i \theta r}-1\right) \mathbb{E}(F) r^{-1} G_{0}((i+1) r,(k+1) r] \mu_{\delta}(d r)\right\} \\
\mathbb{E} e^{i \theta A^{\mathcal{R}^{(0, i \delta],((i+1) \delta, \infty]}}(i \delta,(i+1) \delta]} & =\exp \left\{\int_{0}^{\infty}\left(e^{i \theta r}-1\right) \mathbb{E}(F) r^{-1} G_{0}(r,(i+1) r] \mu_{\delta}(d r)\right\} .
\end{aligned}
$$

Therefore, keeping in mind that

$$
A^{\mathcal{R}_{22}}(k \delta,(k+1) \delta]=A^{\mathcal{R}_{22}}(i \delta,(i+1) \delta]=A^{\mathcal{R}_{22}}(0, \delta],
$$

we have

$$
\begin{gathered}
A(i \delta,(i+1) \delta]-2 \int_{0}^{1} v \bar{G}(v) \int_{v}^{\infty} r^{-1} \mu_{\delta}(d r) d v-\int_{0}^{1} \mathbb{E}(F) \bar{G}_{0}(r) \mu_{\delta}(d r) \\
=A(i \delta,(i+1) \delta]-2 \int_{0}^{1} v \bar{G}(v) \int_{v}^{\infty} r^{-1} \mu_{\delta}(d r) d v-\int_{0}^{1} \mathbb{E}(F) G_{0}(r,(i+1) r] \mu_{\delta}(d r) \\
\left.\quad-\int_{0}^{1} \mathbb{E}(F) G_{0}((i+1) r,(k+1) r]\right) \mu_{\delta}(d r)-m_{k}(\delta) \\
=A^{R_{22}}(0, \delta]-m_{k}(\delta)+o_{p}(a(\delta)) .
\end{gathered}
$$


We thus have that

$$
\begin{aligned}
\left(\begin{array}{c}
A(0, \delta] \\
A(\delta, 2 \delta] \\
\vdots \\
A(k \delta,(k+1) \delta]
\end{array}\right) & -\left\{2 \int_{0}^{1} v \bar{G}(v) \int_{r=v}^{\infty} r^{-1} \mu_{\delta}(d r) d v-\int_{0}^{1} \mathbb{E}(F) \bar{G}_{0}(r, \infty] \mu_{\delta}(d r)\right\}\left(\begin{array}{c}
1 \\
1 \\
\vdots \\
1
\end{array}\right) \\
= & \left(\begin{array}{c}
A^{R_{22}}(0, \delta]-m_{k}(\delta) \\
A^{R_{22}}(0, \delta]-m_{k}(\delta) \\
\vdots \\
A^{R_{22}}(0, \delta]-m_{k}(\delta)
\end{array}\right)+\left(\begin{array}{c}
o_{p}(a(\delta)) \\
o_{p}(a(\delta)) \\
\vdots \\
o_{p}(a(\delta))
\end{array}\right)
\end{aligned}
$$

and the conclusion of Proposition 7 follows.

4.2. Correlation structure. Despite the fact that when we go all the way to the limit as $\delta \rightarrow 0$ we get a degenerate dependence structure, for fixed $\delta>0$, decay of correlations over time slots spaced by $k$, as $k \rightarrow \infty$ exhibits long range dependence. In this section we will prove the following result.

Proposition 8. For fixed $\delta>0$, as $k \rightarrow \infty$,

$$
\operatorname{Cov}(A(0, \delta], A(k \delta,(k+1) \delta]) \sim(\text { constant }) \bar{G}_{0}(k) \sim(\text { constant }) k^{-\left(\alpha_{F}-1\right)} L_{F}(k)
$$

and thus the stationary sequence $\{A(k \delta,(k+1) \delta],-\infty<k<\infty\}$ exhibits long range dependence.

Referring to Figure 3, we see we can write

$$
\begin{aligned}
& A(0, \delta]=A^{\mathcal{R}_{11}}(0, \delta]+A^{\mathcal{R}_{12}}(0, \delta]+A^{\mathcal{R}_{22}}(0, \delta]+A^{\mathcal{R}_{21}}(0, \delta]+I_{1} \\
& =\sum_{k:\left(\Gamma_{k}, L_{k}, R_{k}, F_{k}\right) \in \mathcal{R}_{11}} R_{k}\left(\delta-\Gamma_{k}\right)+\sum_{k:\left(\Gamma_{k}, L_{k}, R_{k}, F_{k}\right) \in \mathcal{R}_{12}} R_{k}\left(\delta-\Gamma_{k}\right) \\
& +\sum_{k:\left(\Gamma_{k}, L_{k}, R_{k}, F_{k}\right) \in \mathcal{R}_{22}} R_{k} \delta+\sum_{k:\left(\Gamma_{k}, L_{k}, R_{k}, F_{k}\right) \in \mathcal{R}_{21}} R_{k} \delta+I_{1}
\end{aligned}
$$

and

$$
\begin{aligned}
& A(k \delta,(k+1) \delta]= A^{\mathcal{R}_{11}}(k \delta,(k+1) \delta]+A^{\mathcal{R}_{12}}(k \delta,(k+1) \delta]+A^{\mathcal{R}_{22}}(k \delta,(k+1) \delta]+A^{\mathcal{R}_{21}}(k \delta,(k+1) \delta]+I_{2} \\
&=\sum_{k:\left(\Gamma_{k}, L_{k}, R_{k}, F_{k}\right) \in \mathcal{R}_{11}} R_{k}\left(\Gamma_{k}+L_{k}-\delta k\right)+\sum_{k:\left(\Gamma_{k}, L_{k}, R_{k}, F_{k}\right) \in \mathcal{R}_{12}} R_{k} \delta \\
& \quad+\sum_{k:\left(\Gamma_{k}, L_{k}, R_{k}, F_{k}\right) \in \mathcal{R}_{22}} R_{k} \delta+\sum_{k:\left(\Gamma_{k}, L_{k}, R_{k}, F_{k}\right) \in \mathcal{R}_{21}} R_{k}\left(\Gamma_{k}+L_{k}-k \delta\right)+I_{2},
\end{aligned}
$$

where $I_{1}$ and $I_{2}$ are independent of the other summands and of each other and do not affect covariance calculations. We thus have

$$
\begin{aligned}
& \operatorname{Cov}(A(0, \delta], A(k \delta,(k+1) \delta]) \\
& =\operatorname{Cov}\left(A^{\mathcal{R}_{11}}(0, \delta], A^{\mathcal{R}_{11}}(k \delta,(k+1) \delta]\right)+\operatorname{Cov}\left(A^{\mathcal{R}_{12}}(0, \delta], A^{\mathcal{R}_{12}}(k \delta,(k+1) \delta]\right. \\
& \quad+\operatorname{Cov}\left(A^{\mathcal{R}_{22}}(0, \delta], A^{\mathcal{R}_{22}}(k \delta,(k+1) \delta]\right)+\operatorname{Cov}\left(A^{\mathcal{R}_{21}}(0, \delta], A^{\mathcal{R}_{21}}(k \delta,(k+1) \delta]\right) .
\end{aligned}
$$

The dominant term comes from the region $\mathcal{R}_{22}$ as we now show.

4.2.1. Contribution to the covariance from $\mathcal{R}_{22}$. Since

$$
A^{\mathcal{R}_{22}}(0, \delta]=A^{\mathcal{R}_{22}}(k \delta,(k+1) \delta],
$$

we have

$$
\operatorname{Cov}\left(A^{\mathcal{R}_{22}}(0, \delta], A^{\mathcal{R}_{22}}(k \delta,(k+1) \delta]\right)=\operatorname{Var}\left(A^{\mathcal{R}_{22}}(0, \delta]\right) .
$$


If $P$ is a Poisson distributed random variable independent of summands $\left\{\xi_{n}, n \geq 1\right\}$, then

$$
\operatorname{Var}\left(\sum_{i=1}^{P} \xi_{i}\right)=\mathbb{E}(P) \cdot \mathbb{E}\left(\xi_{1}^{2}\right) .
$$

Therefore, we have

$$
\begin{aligned}
\operatorname{Var}\left(A^{\mathcal{R}_{22}}(0, \delta]\right) & =\iiint_{\substack{s<0, r>0 \\
l>(k+1) \delta+|s|}} r^{2} \delta^{2} \lambda d s F_{L, R}(d l, d r) \\
& =\int_{s=(k+1) \delta}^{\infty} \lambda d s \int_{r=0}^{\infty} r^{2} \delta^{2} \bar{G}(r s) F_{R}(d r) \\
& =\lambda \delta^{2} \int_{r=0}^{\infty} r \bar{G}_{0}((k+1) r \delta) F_{R}(d r) \mathbb{E}(F) \\
& =\lambda \delta \int_{0}^{\infty} r \bar{G}_{0}((k+1) r) F_{R}\left(\delta^{-1} d r\right) \mathbb{E}(F) \\
& =\int_{0}^{\infty} r \bar{G}_{0}((k+1) r) \mu_{\delta}(d r)=:\left(a_{k}^{\#}(\delta)\right)^{2} .
\end{aligned}
$$

Growth RATE OF $\left(a_{k}^{\#}(\delta)\right)^{2}$. We now discuss the growth rate of $\left(a_{k}^{\#}(\delta)\right)^{2}$ as a function of $k$, keeping $\delta>0$ fixed.

Proposition 9. For any fixed $\delta>0$, as $k \rightarrow \infty$,

$$
\left(a_{k}^{\#}(\delta)\right)^{2}=\int_{0}^{\infty} r \bar{G}_{0}((k+1) r) \mu_{\delta}(d r) \sim \bar{G}_{0}(k) \int_{0}^{\infty} r^{2-\alpha_{F}} \mu_{\delta}(d r)
$$

where the constant on the right side is finite.

Proof. It is no loss of generality to suppose for convenience that $\delta=1$ and to neglect $F_{R}(1)$ in the denominator of $\mu_{\delta}$. Then we must show

$$
\int_{0}^{\infty} r \frac{\bar{G}_{0}(k r)}{\bar{G}_{0}(k)} F_{R}(d r) \rightarrow \int_{0}^{\infty} r^{2-\alpha_{F}} F_{R}(d r) .
$$

By Fubini's theorem, the left side is equal to

$$
\int_{0}^{\infty}\left(\int_{r=0}^{s} r F_{R}(d r)\right) \frac{\bar{G}(k s) k}{\mathbb{E}(F) \bar{G}_{0}(k)} d s .
$$

Since

it suffices to show that

$$
\bar{G}_{0}(k) \sim \frac{k \bar{G}(k)}{\mathbb{E}(F)\left(\alpha_{F}-1\right)}
$$

$$
\int_{0}^{\infty}\left(\int_{r=0}^{s} r F_{R}(d r)\right) \frac{\bar{G}(k s)}{\bar{G}(k)} d s \cdot\left(\alpha_{F}-1\right)
$$

converges to the right side of (4.15).

Break the integral in (4.16) into an integration on $[0,1]$ and one on $(1, \infty)$. For $s>1$, we have by Potter's bounds, that for any small $\eta>0$, for all sufficiently large $k$, and some constant $c$,

$$
\frac{\bar{G}(k r)}{\bar{G}(k)} \leq c r^{-\alpha_{F}+\eta} \text {. }
$$

On $[1, \infty)$, the integrand is bounded by

$$
\mathbb{E}(R) c r^{-\alpha_{F}+\eta}
$$


which is integrable on $[1, \infty]$. Thus, by dominated convergence we may integrate to the limit on $[1, \infty)$. On $[0,1]$ the integrand in (4.16) is bounded (neglecting constants) by

Since Karamata's theorem implies

$$
s \frac{\bar{G}(k s)}{\bar{G}(k)} \rightarrow s^{1-\alpha_{F}} .
$$

$$
\int_{0}^{1} s \frac{\bar{G}(k s)}{\bar{G}(k)} d s \rightarrow \int_{0}^{1} s^{1-\alpha_{F}} d s=\frac{1}{2-\alpha_{F}},
$$

the desired result follows from Pratt's lemma (Pratt (1960) or Resnick (1998, page 164)).

4.2.2. Contribution to the covariance from other terms. We now show the contribution to the covariance from the other three terms in (4.14) is $o\left(\bar{G}_{0}(k)\right)$. We use the following formula in our verifications: If $P$ is a Poisson distributed random variable independent of the iid sequence $\left\{\left(\xi_{n}, \eta_{n}\right), n \geq 1\right\}$, then

$$
\operatorname{Cov}\left(\sum_{i=1}^{P} \xi_{i}, \sum_{i=1}^{P} \eta_{i}\right)=\mathbb{E}(P) \cdot \mathbb{E}\left(\xi_{1} \eta_{1}\right) .
$$

CONTRIBUtion FROM $\mathcal{R}_{11}$. Evaluating the expression on the right of (4.17) for the region $\mathcal{R}_{11}$ yields the following formula:

$$
\iiint_{\substack{0<s \leq \delta \\ r>0 \\ k \delta<l+s \leq(k+1) \delta}} r^{2}(\delta-s)(s+l-k \delta) \lambda d s F_{L, R}(d l, d r) \leq \lambda \delta^{2} \int_{s=0}^{\delta} \int_{\substack{k \delta-s<l \leq k \delta+\delta-s \\ r>0}} r^{2} d s F_{L, R}(d l, d r)
$$

(since on the region of integration, both $(\delta-s)$ and $(s+l-k \delta)$ are bounded by $\delta$ )

$$
\begin{aligned}
& =\lambda \delta^{2} \int_{s=(k-1) \delta}^{k \delta} \int_{r>0} r^{2} \int_{s r<u \leq(\delta+s) r} G(d u) F_{R}(d r) \\
& \left.=\lambda \delta^{2} \int_{s=(k-1) \delta}^{k \delta} \int_{r>0} r^{2} G(s r,(\delta+s) r]\right) F_{R}(d r) \\
& \leq \lambda \delta^{2} \int_{s=(k-1) \delta}^{k \delta} \int_{r>0} r^{2} \bar{G}(s r) F_{R}(d r) \\
& \left.=\lambda \delta^{2} \int_{r>0} r G_{0}((k-1) r \delta, k r \delta]\right) \mathbb{E}(F) F_{R}(d r) \\
& \left.=\int_{r>0} r G_{0}((k-1) r, k r]\right) \mathbb{E}(F) \mu_{\delta}(d r) \\
& =o\left(\bar{G}_{0}(k)\right),
\end{aligned}
$$

where the last assertion follows from the analysis of $a_{k}^{\#}(\delta)$ as being of order $\bar{G}_{0}(k)$.

Contribution From $\mathcal{R}_{12}$. The expression on the right of (4.17) gives for the region $\mathcal{R}_{12}$ the following formula:

$$
\begin{aligned}
\iiint_{\substack{0<s \leq \delta \\
r>0 \\
s+l>(k+1) \delta}} r^{2} \delta(\delta-s) \lambda d s F_{L, R}(d l, d r) & =\lambda \delta \int_{s=0}^{\delta} \int_{\substack{r>0 \\
l>k \delta+s}} r^{2} s d s F_{L, R}(d l, d r) \\
& \leq \lambda \delta^{2} \int_{s=0}^{\delta} \int_{\substack{r>k>0 \\
l>k+s}} r^{2} d s F_{L, R}(d l, d r) \\
& =\lambda \delta^{2} \int_{s=k \delta}^{(k+1) \delta} \int_{r>0} \bar{G}(r s) d s r^{2} F_{R}(d r) \\
& =\int_{r>0} r G_{0}(k r,(k+1) r] \mathbb{E}(F) \mu_{\delta}(d r)
\end{aligned}
$$




$$
=o\left(\bar{G}_{0}(k)\right) .
$$

In a similar way we show the contribution from the region $\mathcal{R}_{21}$ is $o\left(\bar{G}_{0}(k)\right)$. This completes the verification of Proposition 8.

\section{Concluding Remarks.}

Our model does a sound job of explaining empirically observed facts we have termed stylized facts. For a fixed time slot, there is observable Gaussian behavior for cumulative input as the rate increases, and the slot width decreases. This Gaussian behavior is on a spatial scale $a(\delta) \rightarrow \infty$, and is responsible for most of the traffic volume. Hence this component can model what in (Sarvotham et al. (2005)) is called $\beta$-traffic. The spatial scaling obscures heavy tailed behavior approximated by infinitely divisible random variables with heavy tails. This heavy-tailed component that disappears in the limit is what generates, for finite scale $\delta$, the bursty behaviour and seems to be the right candidate to model the $\alpha$-traffic component. Sarvotham et al. (2005)) point out that as the aggregation increases the traffic becomes more and more Gaussian which implies the Gaussian character is dominant over the bursty character. In addition for a fixed slot length, dependence across time slots exhibits long range dependence, and this dependance is carried mostly by the Gaussian part, i.e. by the $\beta$-component.

Finally, the model offers a reasonable match to what experimenters actually measure and it would be no problem to simulate such a model. It seems to offer numerous positive features.

As the slot length goes to 0 , the centered and scaled sequence of inputs in successive slots converges to a perfectly correlated limiting Gaussian sequence. This is a consequence of shrinking slot width and is not surprising. The higher the frequency of sampling, the more correlation is to be expected.

Statistical evidence about the dependence structure appropriate for $\left(R_{k}, L_{k}, F_{k}\right)$ is still inconclusive and a more focussed effort on data analysis is warranted. We also intend to assume other dependence structures in our modeling in order to investigate how the conclusions change. In particular the $L R$-model where $\left(L_{k}, R_{k}\right)$ are assumed independent is on our radar. Perhaps a mixture of the $L R$ and $F R$-models is also worthwhile to consider.

In this paper we have focussed on independence of $F$ and $R$. In statistical investigations, these quantities have only been found to satisfy some sort of asymptotic independence (Campos et al. (2005), Heffernan and Resnick (2005)). We have assumed the stronger assumption of independence because we presume an asymptotic form of the property would introduce greater technical complexity without changing the conclusions.

A series of conversations with Rudolf Riedi, Rice University, helped arouse interest in the problems of modelling bursty traffic and led to an incubation of ideas. In particular, Professor Riedi's co-authored paper Sarvotham et al. (2005), a brilliant empirical study of traffic rates, stimulated many of our ideas. Also, a conversation with Professor Christian Houdré, School of Mathematics, Georgia Institute of Technology was helpful in initiating the idea of letting slot length go to zero.

\section{REFERENCES}

M. Arlitt and C.L. Williamson. Web server workload characterization: The search for invariants (extended version). In Proceedings of the ACM Sigmetrics Conference, Philadelphia, Pa, 1996. Available from $\{$ mfa126,carey\}@cs.usask.ca.

N. Ben Azzouna, F. Clérot, C. Fricker, and F. Guillemin. A flow-based approach to modeling adsl traffic on an ip backbone link. Annals of telecommunications, 59(11-12), 2004. Traffic engineering and routing.

L. Breiman. On some limit theorems similar to the arc-sin law. Theory Probab. Appl., 10:323-331, 1965.

F. H. Campos, J.S. Marron, C. Park, S.I. Resnick, and K. Jaffay. Extremal dependence: Internet traffic applications. Stochastic Models, 21(1):1-35, 2005.

D.E. Duffy, A.A. McIntosh, M. Rosenstein, and W. Willinger. Analyzing telecommunications traffic data from working common channel signaling subnetworks. In M.E. Tarter and M.D. Lock, editors, Computing Science and Statistics Interface, Proceedings of the 25th Symposium on the Interface, volume 25, pages 156- 165, San Diego, California, 1993. 
C.A. Guerin, H. Nyberg, O. Perrin, S.I. Resnick, H. Rootzen, and C. Stărică. Empirical testing of the infinite source poisson data traffic model. Stochastic Models, 19(2):151-200, 2003.

D. Heath, S.I. Resnick, and G. Samorodnitsky. Heavy tails and long range dependence in on/off processes and associated fluid models. Math. Oper. Res., 23(1):145-165, 1998. ISSN 0364-765X.

J.E. Heffernan and S.I. Resnick. Hidden regular variation and the rank transform. Adv. Appl. Prob., 37(2):393-414, 2005.

I. Kaj and M.S. Taqqu. Convergence to fractional brownian motion and to the telecom process: the integral representation approach. Available at Department of Mathematics, Uppsala University, U.U.D. M. 2004:16, 2004.

O. Kallenberg. Random Measures. Akademie-Verlag, Berlin, third edition, 1983. ISBN 0-12-394960-2.

T. Konstantopoulos and S.J. Lin. Macroscopic models for long-range dependent network traffic. Queueing Systems Theory Appl., 28(1-3):215-243, 1998. ISSN 0257-0130.

W.E. Leland, M.S. Taqqu, W. Willinger, and D.V. Wilson. Statistical analysis of high time-resolution ethernet Lan traffic measurements. In Proceedings of the 25th Symposium on the Interface between Statistics and Computer Science, pages 146-155, 1993.

W.E. Leland, M.S. Taqqu, W. Willinger, and D.V. Wilson. On the self-similar nature of Ethernet traffic (extended version). IEEE/ACM Transactions on Networking, 2:1-15, 1994.

J. Levy and M. Taqqu. Renewal reward processes with heavy-tailed interrenewal times and heavy-tailed rewards. Bernoulli, 6:23-44, 2000.

K. Maulik and S. Resnick. The self-similar and multifractal nature of a network traffic model. Stochastic Models, 19 (4):549-577, 2003.

K. Maulik, S.I. Resnick, and H. Rootzén. Asymptotic independence and a network traffic model. J. Appl. Probab., 39(4):671-699, 2002. ISSN 0021-9002.

T. Mikosch, S.I. Resnick, H. Rootzén, and A.W. Stegeman. Is network traffic approximated by stable Lévy motion or fractional Brownian motion? Ann. Applied Probability, 12(1):23-68, 2002.

J. Neveu. Processus ponctuels. In École d'Été de Probabilités de Saint-Flour, VI-1976, pages 249-445. Lecture Notes in Math., Vol. 598, Berlin, 1977. Springer-Verlag.

K. Park and W. Willinger. Self-similar network traffic: An overview. In K. Park and W. Willinger, editors, Self-Similar Network Traffic and Performance Evaluation, pages 1-38. Wiley-Interscience, New York, 2000.

J.W. Pratt. On interchanging limits and integrals. Ann. Math. Statist., 31:74-77, 1960.

S.I. Resnick. Extreme Values, Regular Variation and Point Processes. Springer-Verlag, New York, 1987.

S.I. Resnick. Adventures in Stochastic Processes. Birkhäuser, Boston, 1992.

S.I. Resnick. A Probability Path. Birkhäuser, Boston, 1998.

S.I. Resnick. Modeling data networks. In B. Finkenstadt and H. Rootzen, editors, SemStat: Seminaire Europeen de Statistique, Exteme Values in Finance, Telecommunications, and the Environment, pages 287-372. Chapman-Hall, London, 2003.

S.I. Resnick. On the foundations of multivariate heavy tail analysis. In J. Gani and E. Seneta, editors, Stochastic Methods and their Applications, pages 191-212. Applied Probability Trust, 2004. J. Applied Probability Special Volume 41A; Papers in honour of C.C. Heyde.

S.I. Resnick and H. Rootzén. Self-similar communication models and very heavy tails. Ann. Applied Probability, 10: 753-778, 2000.

R. H. Riedi and W. Willinger. Toward an improved understanding of network traffic dynamics. In Self-Similar Network Traffic and Performance Evaluation. Wiley, 2000.

S. Sarvotham, R. Riedi, and R. Baraniuk. Network and user driven on-off source model for network traffic. Preprint. Department of Electrical and Computer Engineering, Rice University, Houston, Texas. To appear: Special Issue of the Computer Network Journal on "Long-range Dependent Traffic", 2005.

M.S. Taqqu, W. Willinger, and R. Sherman. Proof of a fundamental result in self-similar traffic modeling. Computer Communications Review, 27:5-23, 1997.

W. Willinger. Data network traffic: heavy tails are here to stay. Presentation at Extremes-Risk AND SAFETy, Nordic School of Public Health, Gothenberg Sweden, August 1998.

W. Willinger and V. Paxson. Where mathematics meets the Internet. Notices of the American Mathematical Society, 45(8):961-970, 1998.

W. Willinger, M.S. Taqqu, M. Leland, and D. Wilson. Self-similarity in high-speed packet traffic: analysis and modelling of ethernet traffic measurements. Statistical Science, 10:67-85, 1995.

W. Willinger, M.S. Taqqu, M. Leland, and D. Wilson. Self-similarity through high variability: statistical analysis of ethernet lan traffic at the source level (extended version). IEEE/ACM Transactions on Networking, 5(1):71-96, 
1997.

W. Willinger, V. Paxson, and M.S. Taqqu. Self-similarity and heavy tails: Structural modeling of network traffic. In Adler, Robert J. (ed.) et al., A practical guide to heavy tails. Statistical techniques and applications. Boston: Birkhuser. 27-53 . 1998.

Eurandom, Laplace Gebouw 1.09, Den Dolech 2, 5612 AZ Eindhoven, The Netherlands E-mail address: bdauria@eurandom.tue.nl

Sidney Resnick, School of Operations Research and Industrial Engineering, Cornell University, Ithaca, Ny 14853

E-mail address: sir1@cornell.edu 